\title{
ATENDIMENTO MULTIPROFISSIONAL AO PACIENTE HIPERTENSO
}

\author{
MULTIPROFESSIONAL APPROACH TO HYPERTENSIVE PATIENTS
}

Paulo César B.Veiga Jardim¹, Ana Luiza Lima Sousa², Estelamaris Tronco Monego²

Docentes das Faculdades de Medicina ${ }^{1}$ e de Enfermagem e Nutrição ${ }^{2}$ da Universidade Federal de Goiás.

CORRESPONDÊNCIA: Liga de Hipertensão Arterial Hospital das Clínicas das Faculdades de Medicina, Enfermagem e Nutrição da Universidade Federal de Goiás. Rua 115-F no 135 - Setor Sul - CEP: 74085-300 - Goiânia - GO

JARDIM PCBV; SOUSA ALL \& MONEGO ET. Atendimento multiprofissional ao paciente hipertenso. Medicina, Ribeirão Preto, 29: 232-238, abr./set. 1996.

RESUMO: Os maiores problemas do tratamento da hipertensão arterial estão relacionados com a observância às orientações instituídas, sejam elas medicamentosas ou não. $O$ atendimento dos hipertensos por diferentes profissionais, trabalhando conjuntamente melhora, em muito, a adesão à terapêutica recomendada. A alternativa mais adequada para este tipo de atuação é a formação de equipes multiprofissionais. A composição de uma equipe multiprofissional pode ser efetivada em qualquer serviço de saúde. Do grupo poderão fazer parte todos os indivíduos existentes no serviço e que tenham contato com os pacientes (médicos, enfermeiros, nutricionistas, assistentes sociais, psicólogos, professores de Educação Física e funcionários administrativos). Para a formação da equipe, não é necessário contar com todos os tipos de profissionais, mas aqueles disponíveis em cada local, desde que estejam imbuídos dos mesmos ideais de atendimento conjunto. É importante a unidade de atuação e o treinamento adequado. As tarefas de cada um são específicas, mas em diversos momentos ocorre um imbricamento natural de funções o que será revertido em benefício dos pacientes. Estes, sendo submetidos a um volume muito maior de informações sobre seus males e como evitá-los, acabarão por incorporar hábitos mais adequados e serão, efetivamente, elementos ativos no seu tratamento. Vale por fim destacar que este tipo de atuação propiciará aos indivíduos uma assistência de melhor qualidade, não só no caso da hipertensão mas também na abordagem de qualquer tipo de patologia médica.

UNITERMOS: Hipertensão. Equipe de Assistência ao Paciente. Educação em Saúde.

\section{INTRODUÇÃO}

A hipertensão arterial representa importante fator de risco cardiovascular e pela sua alta prevalência constitui problema de saúde pública em todo o mundo, inclusive em nosso país ${ }^{\mathbf{1 , 2}}$. É hoje uma das principais causas das licenças médicas e, também, de aposentadoria precoce.

Seu diagnóstico é extremamente simples e barato. No arsenal terapêutico, as medidas para o trata- mento não medicamentoso são bastante conhecidas e eficazes. $\mathrm{O}$ tratamento medicamentoso teve grande avanço no decorrer das últimas décadas e, atualmente, contamos com medicamentos bastante eficazes e com poucos efeitos colaterais $\mathbf{s}^{\mathbf{3 , 4}, 5}$. Apesar disto, estamos longe de conseguir níveis satisfatórios de sucesso no controle da hipertensão arterial. Um ponto de estrangulamento, tem sido a falta de adesão ao tratamento que é, aliás, fator crucial para se obter uma resposta adequada a qualquer tipo de orientação ${ }^{6,7}$. 
A hipertensão tem origem multicausal e multifatorial, o meio ambiente exerce influência na sua gênese. Hábitos de vida inadequados são fortes determinantes de valores elevados da pressão arterial. O tratamento inicial da hipertensão envolve uma abordagem não medicamentosa e um aspecto primordial envolve, obrigatoriamente, modificações destes hábitos.

Modificar hábitos de vida é interferir na qualidade de vida das pessoas, na sua forma de viver e, em última instância, na sua própria concepção de SAÚDE. O conceito de saúde de cada um é formado a partir de toda a sua vivência, participando de sua constituição a formação familiar, as relações de trabalho, as relações sociais, enfim toda a sua cultura.

Os profissionais de saúde, de uma maneira geral, têm formação muito específica e, além disto, não têm treinamento que fuja ao tecnicismo de sua área. Agindo isoladamente ficam muito limitados no que tange a estes aspectos e o resultado final é uma pobre adesão a qualquer tipo de orientação, fazendo com que um grande número de pacientes abandone o tratamento, precocemente. Outro ponto crítico na adesão é dado pelo grupo de pacientes, apesar de, teoricamente, estarem sob tratamento não seguem a orientação prescrita. Resta, então, um pequeno número que é fiel à terapêutica instituída.

Sabemos que ao assumir, corretamente, o tratamento não medicamentoso (chamado não farmacológico ou mudanças do estilo de vida) o indivíduo está dando o passo mais importante para a obtenção do sucesso quanto ao controle de sua pressão. Isto porque esta primeira fase (tratamento não medicamentoso), que é obrigatória a todos, nos dará a medida exata do comprometimento do indivíduo com sua saúde. Só o paciente consciente da importância do tratamento fará uso correto da medicação quando esta for necessária.

A conscientização está intimamente relacionada ao grau de informação que o paciente incorporou sobre os riscos para sua saúde e, mais uma vez, existem limitações para que estas informações sejam transmitidas por um único profissional de saúde $\mathbf{8 , 9 , 1 0 , 1 1}$.

O médico em particular, por um desvio de formação, está muito pouco treinado para dispender tempo com seu paciente, passando-lhe informações detalhadas sobre hábitos de vida. A linguagem que utiliza e a própria postura que adota de distanciamento são outras barreiras para a comunicação. O sistema de saúde funciona de maneira crítica, fazendo com que, na grande maioria dos serviços, haja o atendimento de um número excessivo de casos o que restringe ainda mais a atenção do médico ao paciente. Todos estes aspectos tornam ainda mais difícil a passagem de informações aos pacientes, limitando sua atuação em aspectos que são erroneamente considerados secundários.

Objetivos múltiplos exigem, diferentes abordagens e a formação de uma equipe multiprofissional irá proporcionar esta ação diferenciada, vindo a corrigir a grande limitação que temos no tratamento dos pacientes hipertensos ${ }^{\mathbf{9 , 1 0 , 1 1}}$.

Diante da ação da equipe multiprofissional, o paciente será submetido a uma gama muito maior de informações com enfoques diferentes e teremos, então, com um indivíduo bem orientado uma boa adesão, com a possibilidade da incorporação de hábitos adequados de vida e, quando necessário, do uso correto dos medicamentos prescritos ${ }^{\mathbf{1 2}, 13,14}$. Por outro lado, o benefício será estendido a toda a equipe de saúde que será muito enriquecida pela troca de informações, reforços mútuos e estímulos trazidos pelo sucesso alcançado.

\section{A EQUIPE MULTIPROFISSIONAL}

Na composição da equipe multiprofissional, pressupõe-se a participação ativa do paciente. Este deve ser considerado como o elemento número um, pois representa o próprio sentido da formação do grupo. Sua inclusão, como elemento integrante da equipe, cria com o mesmo compromissos para com o sucesso da terapêutica, tornando-o sujeito e não simples objeto das ações de saúde a ele dirigidas ${ }^{11}$.

Os demais elementos serão aglutinados a partir de um princípio básico que será o de alcançar metas bastante objetivas que, no caso da hipertensão, são representadas pela obtenção de mudanças no estilo de vida dos pacientes, disciplina no sentido do uso de medicamentos, quando necessários, e comparecimento pontual aos agendamentos.

Os elementos que vão compor a equipe serão todos aqueles que, fazendo parte de um determinado serviço de saúde, estejam envolvidos nos cuidados aos pacientes e imbuídos do mesmo espírito que deve ser sempre o de propiciar melhor atenção ao paciente.

A equipe multiprofissional poderá ser constituída de médicos, enfermeiros, auxiliares de enfermagem, atendentes, nutricionistas, psicólogos, assistentes sociais, professores de Educação Física e funcionários administrativos, etc. 
É importante a agregação de pessoas que pratiquem a mesma filosofia de trabalho e por isso coloquem acima de interesses pessoais (posição, poder, etc.) o bem estar do paciente e o sucesso no tratamento preconizado.

O que deve direcionar o funcionamento do grupo é a harmonia de objetivos e ações compatíveis com as metas que se pretende alcançar. Trabalhar em equipe não significa a existência de pessoas trabalhando ao mesmo tempo em um espaço físico, mas sim trabalhar lado a lado, conhecendo o papel que cada um desempenha e sabendo da importância que cada um tem no desenvolvimento do projeto como todo.

Em cada momento, em cada ação e em cada setor, um elemento terá sua participação mais ou menos destacada e, respeitadas as especificidades profissionais, todos terão o mesmo peso para o sucesso da empreitada.

As grandes vantagens deste tipo de atuação são:

a) o número de pessoas atendidas será maior e tão maior quanto mais afinada estiver a equipe nos seus diversos modos de abordagem;

b) a adesão ao tratamento será nitidamente superior;

c) conseqüentemente, teremos um número muito maior de pacientes com a pressão arterial sob controle;

d) cada paciente poderá ser um replicador de conhecimentos sobre hábitos saudáveis de vida.

As ações poderão ser individuais e/ou coletivas, ou seja, os pacientes poderão sofrer interferências em caráter pessoal, no consultório ou, então, participar de trabalhos de grupo que podem ser desenvolvidos em qualquer local do Serviço, ou mesmo fora dele.

\section{A FORMAÇÃO DA EQUIPE}

Nossa experiência tem demonstrado que o primeiro aspecto a ser considerado é a presença de indivíduos que tenham interesse neste tipo de atuação ${ }^{11}$. Devemos constituir um grupo onde a filosofia norteadora seja o trabalho coletivo e nunca os caprichos individuais. A identificação de pessoas com este perfil será capital no nosso sucesso.

Como afirmamos anteriormente, todos os elementos que sejam ativos nos serviços poderão fazer parte do grupo. Em cada local, de acordo com a disponibilidade dos profissionais poderemos constituir uma equipe diferente, mas atuando com a mesma eficiência.

Em nosso caso, temos um grupo constituído de médicos, enfermeiros, auxiliares e técnicos de enfermagem, nutricionistas, professores de Educação Física e funcionários administrativos além de alunos da graduação e pós-graduação ${ }^{10,11,12,13,14}$. Existem outros serviços criados sob nossa orientação que contam com médicos, técnicos de enfermagem e funcionários administrativos, tendo se mostrado, também, bastante eficientes no tratamento dos hipertensos.

O trabalho deve ser iniciado buscando uma uniformização de linguagem, evitando-se assim idéias conflitantes que possam dificultar o processo educativo do paciente. Cada elemento tem o papel de educador em saúde e sua ação no evoluir do trabalho é bastante definida, havendo ocasionalmente um imbricamento de funções que podem ocorrer, naturalmente, como forma de fortalecimento das ações que se buscam.

O treinamento inicial para os procedimentos técnicos deve ser enfatizado e as correções de rumo devem ser estimuladas em reuniões de equipe realizadas, periodicamente.

Assim, quando vai ser formada uma nova equipe devemos cumprir algumas etapas, como se segue:

a) identificar elementos que comunguem da mesma filosofia de trabalho;

b) identificar elementos que, potencialmente, possam ser integrados neste tipo de atividade;

c) traçar metas (a curto, médio e longo prazo) a serem alcançadas;

d) estabelecer um cronograma de atuação;

e) estabelecer prioridades de ações;

f) iniciar o treinamento da equipe para uniformização de condutas;

g) organizar sistema de informação para retroalimentar o sistema, isto poderá ser feito através de reuniões periódicas, boletins, relatórios, etc.

\section{A AÇÃO DE CADA PROFISSIONAL}

Apesar de haver uma definição bastante clara do papel que será exercido por cada profissional, pela própria especificidade de cada um deles, haverá diversos momentos em que as funções serão comuns de maneira, absolutamente, natural. 


\section{AÇÕES INDIVIDUAIS}

Em alguns serviços, não haverá alguns dos profissionais aqui listados, isto porém não deve ser motivo para a não formação da equipe, basta neste caso haver a substituição de um elemento por outro.

\section{A - Enfermagem}

1. Pré-consulta (triagem/confirmação diagnóstica);

2. Consulta de enfermagem (CE) - para pacientes com PA sob controle);

3. Pós-consulta (reforço de orientações após as CM);

4. Reencaminha ao médico, anualmente, ou caso surja alguma alteração;

5. Encaminha à Nutrição, quando necessário;

6. Encaminha ao Serviço Social, quando necessário;

7. Encaminha à Psicologia, quando necessário;

8. Controle dos retornos;

9. Contato e busca dos faltosos (aerograma e/ou visita domiciliar);

10. Coordenação do serviço;

11. Projetos de pesquisa.

\section{B - Medicina}

1. Consulta médica inicial (CM);

2. Consultas médicas de seguimento $(\mathrm{CM})$ até controle da PA;

3. Encaminha à Enfermagem(CE), quando a PA estiver sob controle;

4. Encaminha à Nutrição, quando necessário (grandes obesos, dislipêmicos, diabéticos rebeldes, etc);

5. Encaminha à Psicologia;

6. Encaminha ao Serviço Social, quando necessário;

7. Reconsulta periódica para pacientes com pressão controlada e em intervalos menores, se e quando necessário;

8. Coordenação de Serviço;

9. Projetos de pesquisa.

\section{C - Nutrição}

1. Consulta de Nutrição $(\mathrm{CN})$ para pacientes encaminhados;

2. Encaminha à $\mathrm{CM}$, quando necessário;

3. Encaminha à $\mathrm{CE}$, quando necessário;

4. Encamina ao Serviço Social, quando necessário;
5. Encaminha à Psicologia, quando necessário;

6. Coordenação de Serviço;

7. Projetos de pesquisa.

\section{D - Psicologia}

1. Consulta de Psicologia (individual ou em grupo);

2. Atendimento aos familiares;

3. Reencaminha aos demais profissionais;

4. Coordenação de Serviço;

5. Projetos de pesquisa.

\section{E - Serviço Social}

1. Atendimento aos pacientes;

2. Atendimento aos familiares;

3. Contato e busca aos faltosos (aerograma e visita domiciliar);

4. Coordenaçãa de Serviço;

5. Projetos de pesquisa.

\section{F - Funcionários administrativos}

1. Atendimento aos pacientes;

2. Agendamento das consultas;

3. Agendamento das reuniões (do serviço e dos pacientes);

4. Contato e busca aos faltosos (aerograma e visita domiciliar);

5. Projetos de pesquisa.

\section{G - Professores de Educação Física}

1. Atendimento a pacientes;

2. Caminhadas com pacientes;

3. Encaminha aos demais profissionais.

Algumas destas ações são óbvias e estão inseridas na atuação própria de cada profissional. Outras, podem ser exercidas por vários dos elementos do grupo, dependendo da disponibilidade e existência de cada um no local.

As ações da enfermagem ${ }^{15,16}$ são muito importantes e se iniciam com a pré-consulta. Neste procedimento, é feito o primeiro contato do paciente com o Serviço e ocorre ou não a confirmação do diagnóstico. São colhidas informações sobre fatores de risco cardiovascular, conhecimento sobre hipertensão e do- 
enças cardiovasculares, fatores hereditários, hábitos alimentares, etc. Nos casos em que não se confirme o diagnóstico de HA, são feitas orientações quanto a hábitos de vida e fatores de risco cardiovascular, havendo a recomendação de retorno anual. Na presença de importantes fatores de risco (grandes obesos, tabagistas, etc.) os indivíduos são convidados para participar das atividades educativas, em grupo. Quando se confirma a HA, a enfermagem faz a primeira orientação sobre mudanças no estilo de vida (Tratamento não Medicamentoso) e agenda o paciente para Consulta Médica em um espaço de tempo o mais curto possível. Caso os níveis pressóricos sejam muito elevados, a Consulta Médica será agendada com prioridade. É importante ressaltar que o intervalo entre a pré-consulta e a primeira consulta médica não deve ser muito longo, mesmo que os níveis de pressão não sejam alarmantes, isto porque quando este espaço de tempo é maior, ocorre evasão do futuro paciente, que nem chega a vir à primeira consulta. Uma estratégia que utilizamos, quando a pletora de consultas médicas estiver dificultando o agendamento, é marcar uma Consulta de Enfermagem em espaço de tempo mais curto para haver uma agregação do indivíduo ao tratamento.

Outro momento de atuação da enfermagem é a Consulta de Enfermagem propriamente dita. São agendados para este procedimento os indivíduos que, após atendimentos pelo médico, têm sua pressão controlada. O seguimento passa a ser feito pela enfermeira que será a responsável pela manutenção do tratamento. O novo encaminhamento ao serviço médico será feito em caso de necessidade (perda de controle da PA, aparecimento de intercorrências, etc) ou a intervalos periódicos. Em estudo realizado por nós, observamos que o tempo adequado para o reencaminhamento à Consulta Médica é de 9 meses ${ }^{16}$. Após este intervalo, começa a haver escape no controle da pressão e perda da adesão ao tratamento.

A Pós-Consulta de enfermagem ocorre logo após a Consulta Médica e é um momento de reforço de toda a orientação dada pelo médico. Observamos que com esta providência a observância às orientações aumenta, seja quanto às medidas não medicamentosas, seja quanto ao uso adequado da medicação quando prescrita.

A enfermagem poderá fazer encaminhamentos a cada um dos outros profissionais, quando julgar conveniente.

O controle de retornos, contato e busca aos faltosos pode ser feito pela enfermagem ou por vários dos profissionais do grupo (funcionários administrativos, serviço social, etc.) e vai depender da disponibilidade dos mesmos em cada local. A estratégia é variável e pode ser feito por telefone, através do envio de aerogramas e até por visita domiciliar. O contato telefônico é mais simples e prático, entretanto a disponibilidade deste meio é bem mais restrita. A busca, através de aerograma, é muito simples, barata e tem muito boa eficiência em recuperar os faltosos, sendo que em nossa experiência é perfeitamente equiparável à visita domiciliar. Esta última encerra um grande esforço operacional sem vantagens que a justifiquem.

É fundamental para o sucesso de cada um dos métodos, uma secretaria que seja competente na obtenção correta dos endereços dos pacientes, além de pontos de referência adicional ${ }^{14}$. Este fato se prende à grande frequiência com que os pacientes mudam de endereço, dificultando em muito o encontro dos mesmos.

O cartão de aprazamento é vital para o controle dos retornos e descoberta dos faltosos, pode ser simples com controle manual, ou sofisticado, através de computadores nos locais em que seja possível. Pouco importa o método, mas ao fim de cada mês devemos ter o controle dos faltosos, para o registro da sua ausência e início de busca.

As ações médicas são bastante específicas e dispensam detalhamento. Alguns aspectos devem ser ressaltados:

Os encaminhamentos que, porventura, ocorram não tiram do médico a responsabilidade de orientação quanto a hábitos de vida e o tempo gasto com o paciente é fundamental para a coesão da equipe.

As ações dos demais membros da equipe (serviço social, psicologia, professores de Educação Física, administrativos) são, também, bastante claras e dispensam outras especificações.

A Coordenação do Serviço pode ser exercida por vários dos componentes do grupo e vai depender da maior disponibilidade de cada um e, principalmente, da sua permanência por maior período de tempo no local de atuação.

\section{AÇÕES EM GRUPO}

São ações desenvolvidas por todos, às vezes conjuntamente, e às vezes alternadamente (participam vários profissionais, mas não todos de cada vez). 
1) Reuniões de equipe (ao menos quinzenais) para todos;

2) Reuniões com pacientes (quinzenais) - "Clube do Hipertenso" - neste caso, participam alguns, mas não obrigatoriamente todos de cada vez (dependendo do assunto a ser tratado);

3) Projetos de pesquisa;

4) Caminhadas com pacientes (mensais ou até semanais) - participação do professor de Educação Física e alguns outros elementos da equipe (que podem revezar).

Nas reuniões de equipe se busca uma troca de informações quanto ao andamento do trabalho. As arestas são aparadas, são estabelecidas novas metas e retomadas metas antigas. É aqui que "se lava a roupa suja" o que, aliás, é o único modo de se conseguir progresso e tornar o trabalho realmente de equipe.

Não se pode temer as discussões, as opiniões, às vezes divergentes, apenas indicam o interesse geral no sucesso do trabalho e só a troca franca de idéias poderá nos levar a atingir o objetivo maior.

Nas reuniões do "Clube do Hipertenso" são tratados assuntos gerais, principalmente relacionados à Hipertensão Arterial e Fatores de Risco Cardiovascular. São reuniões informais e abertas que ocorrem, em nosso caso, a cada 15 dias. Têm a participação de um ou vários elementos da equipe, dependendo do tema a ser tratado e eventualmente de pessoas convidadas para abordar algum assunto específico. Em diversas ocasiões, são discutidos temas, aparentemente, desvinculados de doenças cardiovasculares, mas por representarem preocupação dos participantes são trazidos à tona e esgotados na troca de informações ${ }^{8}$.

É o interesse de todos que acaba por determinar o rumo das discussões. Há, porém, uma tendência natural das pessoas em sugerir assuntos que acabam sendo muito importantes para o desenvolvimento do grupo.

A convivência em comum estimula a relação social, possibilita a troca de informações e permite o apoio mútuo. O paciente, neste tipo de atividade, identifica-se com outros pacientes com problemas semelhantes, aprendendo a expressar seus medos e expectativas. Passa a compartilhar das experiências de todos e a discutir, buscando soluções reais para problemas de saúde como os seus.

Nos projetos de pesquisa a participação coletiva é fundamental, pois só assim se alcançará sucesso no desenvolvimento dos mesmos.
Com relação às caminhadas, é evidente que não se busca um condicionamento aeróbico, pois as caminhadas são feitas a grandes intervalos. A meta, neste caso, é promover uma integração social que sirva como estímulo para os que ainda não incorporaram este hábito de vida. Além disto, nestes momentos, o professor de Educação Física terá a oportunidade de corrigir posturas, orientar quanto a trajes e calçados mais adequados, ensinar técnicas de alongamento, entre outras coisas. O paciente deve ser orientado e estimulado a exercer suas atividades físicas de maneira regular, durante os outros dias do mês, em local próximo a sua residência.

\section{CONCLUSÃO}

A constituição de uma verdadeira equipe multiprofissional, integrada, coesa tem condições de exercer uma atividade bastante profícua em prol dos pacientes. Este tipo de estrutura pode ser utilizado para vários tipos de atendimento e, indubitavelmente, traz grandes benefícios à comunidade onde está inserido.

Para os profissionais que nela atuam há grande satisfação pessoal, sensação do dever cumprido e estímulo a uma atuação cada vez mais abrangente.

Em qualquer serviço de saúde, pode ser criada uma equipe multiprofissional, o número de componentes pouco importa, o tipo de profissional de saúde é secundário, o que determina seu bom funcionamento é a filosofia de trabalho, união na mesma direção.

Qualquer unidade de saúde que tenha a intenção de alcançar metas mais arrojadas deverá investir na formação de equipe multiprofissional. Este grupo propiciará atendimento a maior número de pessoas, desafogará o serviço como um todo, dará um atendimento de melhor qualidade e, em particular, permitirá ao médico a atenção maior aos pacientes que necessitem de cuidados específicos.

Nas doenças cardiovasculares, em geral, e na hipertensão em particular, entendemos que é o melhor meio de alcançarmos sucesso no controle dos nossos pacientes. Aumentando o número de indivíduos com a PA, sob controle, modificando efetivamente hábitos de vida inadequados e criando uma mentalidade futurista no sentido de prevenção das doenças cardiovasculares, estaremos, realmente, contribuindo para uma melhor atenção à saúde de nossa população. 
JARDIM PCBV; SOUSA ALL \& MONEGO ET. Multiprofessional approach to hypertensive patients. Medicina,

Ribeirão Preto, 29: 232-238, apr./sept. 1996.

ABSTRACT: One of the most important difficulties in treatment of hypertension is the compliance to treatment. The approach of hypertensive patients by differents professionals improve the compliance to treatment. A good estrategie to achieve this goal is to organize multiprofessional team. All kind of health professional can participate of the team (phisicians, nurses, nutritionists, members of social service, psychologists, physical trainers, administrative staff) but not all are necessary to begin the team. The most important thing is the uniformity of comportament and of information to the patients. This approach improve the compliance and give benefits in concerning high blood pressure and others cardiovascular risk factors.

UNITERMS: Hypertension. Patient Care Team. Health Education.

\section{REFERÊNCIAS BIBLIOGRÁFICAS}

1 - ORGANIZAÇÃO PAN-AMERICANA DA SAÚDE. A hipertensão arterial como problema de saúde comunitária. In: Manual de Normas operacionais para um Programa de Controle nos diferentes níveis de atenção.OPS, Washington, p. 1-68 1986 (Série Paltex, 3).

2 - ACHUTTI AC et al. Controle da hipertensão arterial: uma proposta de integração ensino-serviço. Ministério da Saúde CDCV/NUTES, Rio de Janeiro, 232p.,1993

3 - II CONSENSO BRASILEIRO PARA O TRATAMENTO DA HIPERTENSÃO ARTERIAL. Arq Bras Cardiol 63: 333-347, 1994.

4 - THE FIFTH REPORT OF THE JOINT NATIONAL COMMITTEE ON DETECTION, EVALUATION, AND TREATMENT OF HIGH BLOOD PRESSURE. Arch Intern Med 153: 154-183, 1993.

5 - NATIONAL HIGH BLOOD PRESSURE EDUCATION PROGRAM WORKING Group report on elderly. Hypertension 23: 275-285, 1994.

6 - TAYLOR W; SACKETT D \& HAYNES RB. The problem of compliance with antihypertensise regimens. Drugs 25: 12-18, 1983.

7 - GIORGI DMA et al. Aderência ao tratamento em hipertensão arterial: influência de variáveis estruturais e de estratégias que visem sua melhora. Rev Bras Med Cardiol 4: 167-176,1985.

8 - SOUSA ALL et al. Uma experiência multiprofissional na abordagem ao paciente hipertenso. Arq Bras Cardiol 59:31-35, 1992.
9 - LITVAK $J$ et al. Programa de cooperacion interpaises de la OPS sobre prevencion de la fiebre reumatica. Bol Of Sanit Panam 88: 98-107,1980.

10 - JARDIM PCBV \& SOUSA ALL. Liga de hipertensão arterial, integrando serviço,ensino e pesquisa. Bol Of Sanit Panam 114: 80-84,1993.

11 - JARDIM PCBV \& SOUSA ALL. A Equipe multiprofissional no tratamento do hipertenso. HiperAtivo 2: 3-6, 1993.

12 - JARDIM PCBV et al. Evaluation of compliance to treatment of patients with hypertension in multiprofessional activities. Am J Hypertens 5: 134-A, 1992.

13 - GARCIA A et al. Non-pharmachological treatment of arterial hypertension - 22 months of follow-up by a multiprofessional team. Hypertension 21:46 A,1993.

14 - SOUSA ALL et al. Rate of abandon of the antihypertensive treatment in the context of a multiprofessional service - A 3 year analysis. Am J Hypertens 8: 100A, 1995.

15 - SOUSA ALL \& JARDIM PCBV. A enfermagem e o paciente hipertenso em uma abordagem multiprofissional - Relato de experiência. Rev Latinoam Enferm 2: 5-17,1994.

16 - MARTINS R; SOUSA ALL \& JARDIM PCBV. Nursing followup to hypertensive patients in a multiprofessional Service. Hypertension 21: 46 A,1993.

Recebido para publicação em 16/01/96

Aprovado para publicação em 25/01/96 